\title{
Cardiotoxicity - the first cause of morbidity and mortality in pediatric patients survivors of acute lymphoblastic leukemia
}

\author{
Letitia Elena Radu ${ }^{1,2 *}$, Roxana Corina Sfetea $^{3}$, Constantin Virgiliu Arion ${ }^{1,2}$, Anca \\ Colita $^{1,2}$
}

1. "Carol Davila" University of Medicine and Pharmacy, Pediatrics Department, Bucharest, Romania 2. Fundeni Clinical Institute, Department of Pediatric Hematology and Oncology, Bucharest, Romania 3. "Carol Davila" University of Medicine and Pharmacy, English Department, Bucharest, Romania

\begin{abstract}
Acute lymphoblastic leukemia is the most common hematological malignancy at pediatric age. Cardiotoxicity holds the first place among the causes of morbidity and mortality in these patients. Anthracyclines are cytostatic drugs frequently associated with cardiotoxicity. Early diagnosis of cardiac impairment during the treatment of pediatric patients is extremely important, both for modulating future chemotherapy and for administering cardioprotective agents. Long term monitoring after chemotherapy helps to identify the risk of late cardiotoxicity among cancer survivors. There are several biomarkers, already in use or still under study, which may represent an operator-independent alternative for echocardiography in the diagnosis of cardiotoxicity. In case of cardiac damage, the clinician has options for treating or limiting the progression, either with the use of already approved agents, such as Dexrazoxane, or by administrating other cardioprotective drugs. International experts are still attempting to establish the best algorithm for early detection of cardiotoxicity, as well as the most efficient treatment plan in case of already existing myocardial damage in these patients. We present a review on treatment-related cardiotoxicity, including mechanisms of development, useful biomarkers and treatment options, after carefully analyzing specialty literature.
\end{abstract}

Keywords: acute lymphoblastic leukemia, cardiotoxicity, biomarkers, cardioprotective agents

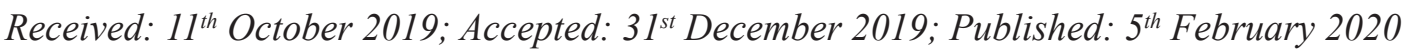

\section{Introduction}

Approximately 130 children/million/year are diagnosed with cancer (1) and $25-30 \%$ of them develop acute leukemia (2). The overall inci- dence of acute lymphoblastic leukemia (ALL) is $30.9 /$ million inhabitants (3). It is mandatory to assign patients to risk groups, to establish the most appropriate therapeutic plan, and to determine the prognosis (4).

*Corresponding author: Letitia Elena Radu, Fundeni Clinical Institute, Department of Pediatric Hematology and Oncology, Bucharest, Romania. E-mail: letitia_radu@yahoo.com 
Currently, an event-free survival of $87.5 \%$ after 5 years has been reached (3). The cardiovascular side effects occupy the first place among the causes of mortality and morbidity (5), more than $7 \%$ of the pediatric oncological patients will develop heart failure after 30 years from the diagnosis (6). The most frequently incriminated agent is the group called anthracyclines (AC). The cardiac damage is dose dependent and a cumulative dose (CD) of $250-300 \mathrm{mg} / \mathrm{m}^{2}$, used by pediatric protocols, usually induces only an asymptomatic pathology, proved by performing echocardiographies (7).

Chemotherapy-induced cardiotoxicity (CIC) can be divided into two categories: type 1, considered irreversible, occurring by damaging the myocytic microstructure, leading to necrosis or cell apoptosis, respectively type 2, represented by myocytic dysfunction without structural anomalies, which can be remitted after the end of the administration of the medicinal product causing it (8).

$\mathrm{AC}$ are frequently used through various pharmaceutical forms: doxorubicin, epirubicin, idarubicin, daunorubicin. The most frequently used product is doxorubicin, extracted from a mutated strain of Streptomyces Peucetius. It is composed of a naftacenequinon nucleus and an amino-sugar, daunozamine. Excretion is done through the liver and the kidneys, the product having a biphasic half-life, after 5 minutes, respectively after 30-40 hours (9). Its structure is presented in Figure 1.

The first documentation of cardiotoxicity in ALL patients was in 1967 (11). AC are known to cause irreversible and dose-dependent cardiac impairment, translated into subclinical dysfunction, arrhythmias, cardiomyopathy, and congestive heart failure (12). There are studies showing patients with partial remission of this injury, probably due to early detection (13).

CIC is defined by the "Cardiac Review and Evaluation Committee" as cardiomyopathy with a decreased left ventricle ejection fraction (LVEF) at a global or mostly septal level, symptoms and signs of congestive heart failure, including galloping noise and tachycardia, a decrease of at least $5 \%$ of LVEF in symptomatic patients or at least $10 \%$ in asymptomatic ones, up to a LVEF value of less than 55\% (14). Another group of criteria used to define CIC involves an increase above the threshold value of troponin I or troponin $\mathrm{T}$, a temporary or persistent decrease of LVEF by $10 \%$ compared to the pretreatment level or a decrease below $55 \%$, new areas of myocardial fibrosis detected by gadolinium-enriched magnetic resonance examination, worsening of diastolic function shown by echocardiography,

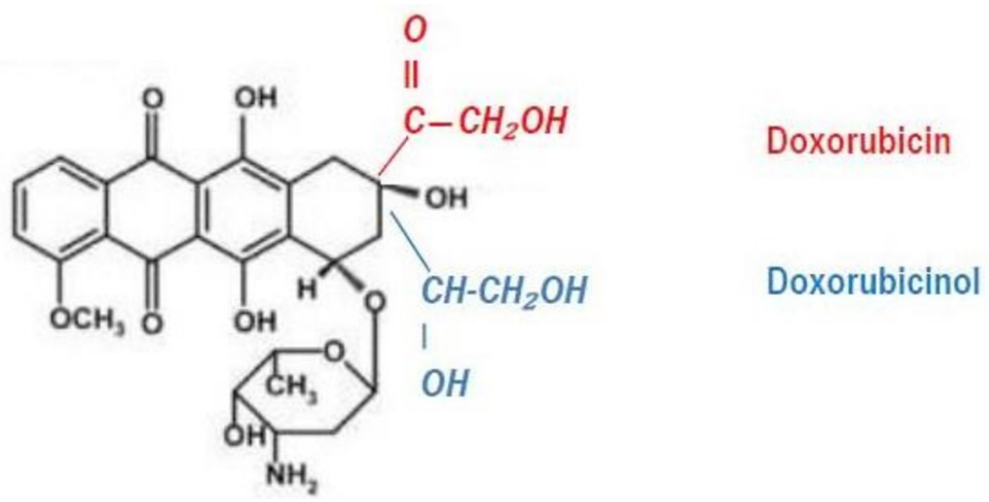

Fig.1. The chemical structure of Doxorubicin and Doxorubicinol (modified and adapted after Wang GX et al (10)) 
pericarditis, newly installed or unstable myocardial ischemia, rhythm disorders, valvulopathies (15).

According to the moment of development, cardiotoxicity is categorized as: acute, during the first week following $\mathrm{AC}$ in fewer than $1 \%$ patients, with arrhythmias, electrocardiogram abnormalities, myocarditis, pericarditis to congestive heart failure, early, during the first year after diagnosis in 1.6-2.1\% patients, with decreased left ventricle contractility, and late, occurring after this temporal interval, $65 \%$ of surviving children presenting cardiac impairment after 6 years from end of therapy (6).

\section{Mechanisms of development}

The mechanisms of CIC include direct cellular toxicity on myocytes, ischemia induced by vasoactive effects, thrombogenesis, myocardial inflammation and interference with transmembrane channels (16). A schematic representation is shown in Figure 2.
One of the hypothesis is the generation of free oxygen radicals following the reaction between the AC structure, oxygen molecules, and cell electrons (18). Another hypothesis is the intermediation of AC drugs among nucleic acids, resulting in the interference of cellular replication through an enzyme-dependent iron-related process, the patients with an increased iron concentration in tissues being prone to cardiac impairment (19). It seems that hemochromatosis C282Y gene carriers present a higher risk for developing CIC (19).

There are two types of topoisomerase 2 (Top2): Top $2 \alpha$, essential in $\mathrm{AC}$ antitumor action, and Top2 $\beta$, whose inhibition causes ruptures in the DNA structure, leading to cellular death. Top2 $\beta$ determines the activation of $\mathrm{p} 53$, stimulates the production of antioxidants, but also inhibits the coactivators $1 \alpha$ and $1 \beta$ of peroxisome activated receptor, which are vital in mitochondrial synthesis. A study on rats showed that an increased blood level of Top $2 \beta$ was noticed in patients sensitive to AC (18).

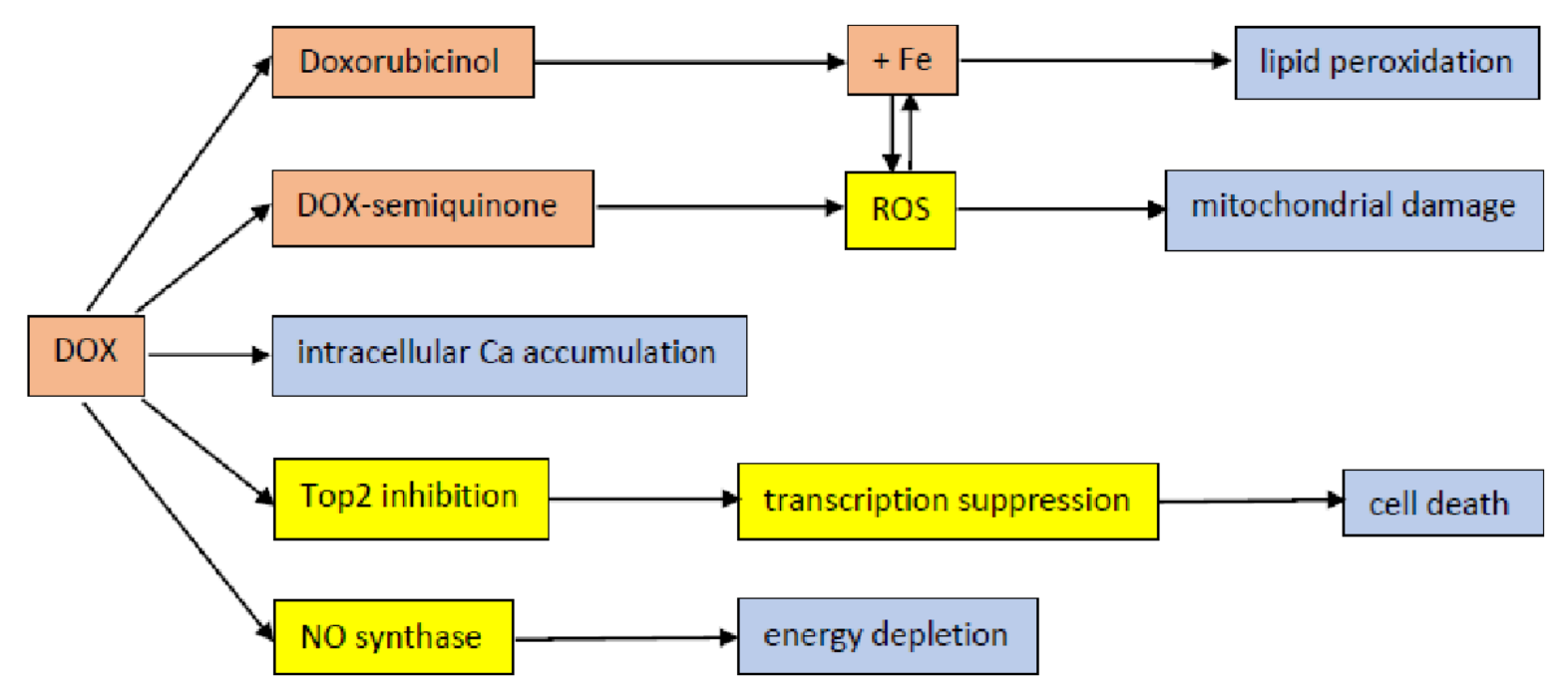

Fig. 2. Mechanisms of action of Doxorubicin at the cellular level.

DOX: doxorubicin, Fe: iron, ROS: reactive oxygen species, Ca: calcium, Top2: topoisomerase, NO: nitric oxide (modified and adapted after Lipshultz SE et al (17)) 
Some experts suggest that dystrophin, a cytoskeletal protein, has a role in the development of CIC, while other scientists study the interaction between sarcolemma, sarcomeres, and the occurrence of both systolic and diastolic cardiac dysfunction (20).

In addition to myocytes, $20 \%$ of the heart structure consists of fibroblasts, endothelial cells, adipocytes, and smooth muscle. They may be affected by AC even before the myocytes, so cardiomyopathy is preceded by alterations in the supporting matrix (20). Moreover, data from animal models suggest that AC leads to a decrease in c-Kit in cardiac progenitor cells, being the reason why the response to pathogenic stress is diminished and the injury repair no longer takes place (21).

Another aspect is the impairment of diastolic relaxation by titin proteolysis, calpain-dependent, and the accumulation of intracellular calcium (22).

Myocytes become more susceptible to changes in neuregulin-1 and ErbB signals, through phosphoinositid-3-kinase, AKT-kinase serin-treonin-specific, mitogen-enabled protein-kinase and ERK-kinase (22). The prolonged exposure of the organism to AC may result in decreased cardiac glutathione peroxidase activity, defects in the respiratory chain with damages to the mitochondrial DNA, and generation of free radicals. They are formed even after completing the AC-based treatment, causing late CIC (6).

Possibly the most important aspect correlated with the occurrence of CIC in ALL pediatric patients is the $\mathrm{CD}$ (6). International guidelines recommend not to exceed $450-550 \mathrm{mg} / \mathrm{m}^{2}$ per patient (18). Exposure to a total dose of 151-200 $\mathrm{mg} / \mathrm{m}^{2} \mathrm{AC}$ is associated with odds ratio of 3.69 of CIC, between $201-250 \mathrm{mg} / \mathrm{m}^{2}$ the risk doubles and the probability reaches 23.47 when the CD increases above $251 \mathrm{mg} / \mathrm{m}^{2}$ (23).

Another risk factor to be considered is gender; girls develop CIC four times more frequently than cancer-diagnosed boys. Obesity also seems to be an important factor (6). Some studies have shown that young age at diagnosis is associated with an increased risk of cardiac impairment (24). Other factors involved in the occurrence of CIC highlight cardiac pathology, metabolic deficits (25), and the type of administered AC product, doxorubicin being more toxic to cardiac tissue than epirubicin (16).

\section{Biomarkers used in diagnosing CIC}

Early diagnosis of cardiac impairment in ALL children is important to modulate chemotherapy and to add cardioprotective agents (25). Distance monitoring after end of treatment is useful to select survivors at risk of developing late cardiotoxic effect (24). At present, the most effective method of detecting myocardial injury has not been established (8).

Differential proteomics is the method which can identify the specific biomarkers of a certain disease/tissue/organ by quantifying the level expressed between the targeted patients and a control group. The term of "biomarker" was defined in 1989 (26). In case of cellular death and metabolic stress, several intracellular proteins are released into the bloodstream (16). In the context of lesions caused by ischemia of myocardial cells, these biomarkers are early indicators of cardiac injury, but CIC is not produced solely through this mechanism; therefore, it is not easy to evaluate the kinetic properties of cardiac markers (27).

Perhaps the most studied tests for early detection of CIC are troponin I, C, and T. These are proteins found in skeletal, myocardial, and smooth muscles (13) and they control the calcium-dependent link between actin and myosin $(28,29)$. In 2004, the FDA called troponins sensitive, specific and robust biomarkers of cardiac injury which enable the detection and quantification of cell lesions and apoptosis caused by drugs (8). In patients with different malignancies, troponin 
values increase directly proportionally to $\mathrm{CD}$ of $\mathrm{AC}$, are correlated with the degree of cardiac impairment found in anatomopathologic analysis, and are associated with clinical signs of left ventricle dysfunction. The levels are even higher in patients subjected to a more aggressive and longer-term chemotherapy regimen (8).

Drafts's study on 37 patients who received doxorubicin, respectively 16 with daunorubicin, showed troponin I had a growing trend, reaching positive values in $25 \%$ patients after six months from the beginning of the treatment (8). Lipshultz et al. followed 100 patients who received doxorubicin and 105 who received doxorubicin associated with dexrazoxane, concluding that the level of troponin I during the first 90 days of treatment was predictable for myocardial damage four years later (8).

A study conducted by Oztarhan on a group of 276 children with ALL, followed over a seven-year period, concluded that diastolic dysfunction appeared earlier compared with the systolic one, respectively to the increase in cardiac biomarkers, as well as the changes in troponin values were associated with the occurrence of congestive myocardial insufficiency (30).

In a reference study, Cardinale et al. showed that in breast cancer patients treated with high doses of chemotherapy there was a clear correlation between the increase in troponin levels after treatment and the decrease in LVEF (31). In another study, Lee analyzed a group of 86 patients and showed troponin values correlated with both LVEF and CD of AC (32).

In opposition, there are studies which contradict the usefulness of these markers and support the hypothesis that the variations are due to physiological changes or underlying cardiovascular conditions (8). Moreover, several studies have shown that troponin was not useful for the assessment of CIC after 1-2 years following chemotherapy (12). Soker and Kervancioglu conducted an analysis on a group of 31 patients treated with
Doxorubicin (33), and Specchia et al. analyzed a group of 17 patients diagnosed with ALL (34), whose level of troponin remained undetectable throughout the study.

In the paper authored by Tian S., reference is made to four studies conducted on breast cancer patients, treated with AC, where the correlation between troponin I and LVEF was analyzed (35). Two of these studies, carried out on 92 and 33 patients, respectively, did not find any correlations $(36,37)$. However, Feola et al. analyzed 53 patients in whom troponin I registered an increase after one month from the beginning of the treatment, which subsequently returned to normal values (38), and Garrone et al. concluded there was a correlation between the increase of this biomarker and the decrease of LVEF in 50 women (39).

In specialty literature, there are few studies on the evaluation of cardiotoxicity in pediatric patients treated with AC by monitoring the dynamic changes in troponin I. In a study on 50 children with ALL, the values of this biomarker changed during therapy (40). However, Rajesh et al. did not find any changes in troponin after treatment in 29 children (8). Furthermore, in a group of 70 patients there were no significant changes (41). Troponin I appears to be more suitable than troponin $\mathrm{T}$ or $\mathrm{C}$ to detect myocardial lesions with greater precision. An explanation for this could be its lower molecular weight and kinetic curve, which enable early quantification (8). The rhythm of troponin release into the bloodstream has not yet been established, so further measurements are needed to capture the exact dynamics (16).

Furthermore, new studies on high-sensitivity troponin are in progress, which appear to offer promising results (42). It was noticed that adding the troponin value to the measurement of global longitudinal deformation led to an increased predictive value, but the echocardiographic parameter remained the only independent predictor within the model (43). 
The natriuretic peptide is synthesized in the atria and ventricles following injury of cardiac walls and increased pressure (28). It produces natriuresis and vasodilation through guanosine-cyclic-monophosphate mediated mechanisms. It can be used in the diagnosis of heart failure and cardiomyopathies (8).

An increase in B-type natriuretic peptide, cleaved from a pre-pro-peptide to pro-BNP (35), is associated with the early detection of cardiac damage in oncologic patients, but it is not correlated with LVEF changes. In parallel, A-type natriuretic peptide does not appear to be useful for this purpose. The inactive fragment of BNP seems to have elevated values in patients diagnosed with cancer, especially in those treated with $\mathrm{AC}(8)$. Moreover, it appears that elevated values of both are predictive for late-onset CIC (13).

The heart-type fatty acid binding protein (H-FABP) is a small molecule found in the cytoplasm, which participates in the elimination of free radical acids. H-FABP records an increase during the first 2-3 hours from the myocardial injury or even after one hour (35).

There are studies attesting the value of H-FABP in the context of patients receiving cytostatic treatment with AC. ElGhandour et al. analyzed H-FABP changes in 40 patients diagnosed with Hodgkin lymphoma who received AC-based treatment, without exceeding $300 \mathrm{mg} / \mathrm{m}^{2} \mathrm{CD}$. The results were favorable, this biomarker increased in $25 \%$ of patients even after the first cycle of chemotherapy, while after the last cycle 8 out of 10 patients had decreased LVEF levels associated with a significant increase in H-FABP (44). Another analysis showed the inhibition of the H-FABP gene expression following the administration of Doxorubicin over a longer period (45). In another study, 20 children who benefited from HSCT were evaluated on days 0,7 , and 21 using a panel of cardiac markers, including H-FABP. It was shown that H-FABP on day 21 was higher than baseline, $\mathrm{p}<0.05$ (46).
A group of 47 adult patients, who received standard chemotherapy or conditioning regimen for HSCT, did not present H-FABP variations (47). The experiment carried out by Lai and Wang divided rabbits into a control group and four groups of subjects which received increased doses of doxorubicin. Only the subset which received the highest CD showed increased values of H-FABP, that is why the authors concluded this biomarker might not be suitable for assessing early cardiac damage induced by AC (48).

high-sensitivity C-reactive protein is a biological marker without cardiac specificity, an acute phase protein, and its expression is adjusted by interleukins 1, 6 and tissue necrosis factor (35). Some international studies have shown that there was a link between elevated values of high-sensitivity C-reactive protein and cancer survivors, but without any correlations with changes in echocardiographic assessment. The motivation seems to be related to the inflammatory effect of chemotherapy and not to the cardiotoxic effect of drugs (8).

Glycogen phosphorylase BB is a newly suggested test for CIC diagnosis (8). It is a heart-specific glycogen-lithic enzyme that carries glucose to smooth muscles (35). In a study related to highdose chemotherapy followed by HSCT, results showed increased levels of this biomarker, not correlated with troponin or natriuretic peptide values, which is why it is not possible to conclude whether it is a more sensitive essay, or the increase is related to the aberrant changes in this protein (8).

Myeloperoxidase is a proatherogenic enzyme present in neutrophils, which leads to the formation of free radicals and lipid peroxidation (49). The increase of the myeloperoxidase value after $\mathrm{AC}$ administration, and its subsequent decrease, proved to be correlated with the occurrence of late cardiac involvement, especially in combination with the modification of echocardiographic parameters (32). 
Circulating antioxidants in peripheral blood appear to register a decrease directly proportionally to the AC dose administrated to patients (8). Circulating microRNAs represent noncoding fractions of RNA and play a role in homeostasis (8) and in the mechanism of response to oxidative stress and cellular injury (50). They can be detected by RT-PCR or sequencing techniques, directly from the tissue or circulating leukocytes (13). The quantification of microRNAs can play a significant role in detecting inflammation and damaged myocardial cells, post-administration of the cytostatic treatment (8).

Galectin-3 is a useful biomarker in detecting myocardial fibrosis in patients with heart failure (13). In studies on patients treated with chemotherapy or cancer survivors, galectin-3 has not demonstrated its role in the early diagnosis of CIC, being uncorrelated with other biological markers with cardiac specificity or with echocardiographic parameters (8).

ST-2 protein is part of the interleukin 1 family and is a marker used in diagnosing myocardial infarction and heart failure. In some studies, this test had increased values when analyzing cancer survivors compared with the control group (13). Nitric oxide is a small molecule, produced from arginine, with an extremely important role in the mechanisms of CIC. A study showed an increased nitric oxide value in pediatric patients treated with AC, correlated with decreases in LVEF (35).

\section{Cardioprotective agents}

Administration of the same dose of AC during a longer period has been discussed. Although it has been shown that infusion over 48-96 hours in female patients undergoing treatment for breast cancer reduces the risk for cardiac impairment, compared with the bolus administration of chemotherapy, in children with ALL there has not been any decrease in the incidence of early or late cardiotoxicity with the same approach (6). Another investigated aspect was the type of AC product used. Even though preclinical studies showed that epirubicin and idarubicin might be less cardiotoxic, in tests on human patients these results were not conclusive (6). There are several new products (liposomal doxorubicin, liposomal daunorubicin, and pegylated liposomal doxorubicin) which have shown the same tumoricidal properties as traditional preparations, but without the association with the same degree of cardiac impairment, toxic CD rising to 600-900 $\mathrm{mg} / \mathrm{m}^{2}$. However, for the time being, these drugs have been studied only on adult groups, with no extrapolation of the results in children (8).

Another strategy to reduce the risk of CIC development is the concomitant administration of cardioprotective agents. Dexrazoxane is the only drug approved by the FDA to prevent the occurrence of cardiotoxicity induced by AC administration (22). It inhibits Top2, leading to the chelation of free iron or doxorubicin-iron complex, thus limiting the formation of free oxygen radicals (6).

Currently there are numerous studies attesting to the cardioprotective value of this drug, both in adult patients and children. By administering it simultaneously with the AC dose, dexrazoxane protects the heart from side effects (6), decreases the level of cardiac biomarkers (8), and improves the results obtained in echocardiographic assessments (50). Likewise, according to studies, it seems that the effect is much more pronounced in female patients than in boys (51).

However, there are opinions against the routine use of dexrazoxane because of possible protective effect not only at myocytic level, but also on the tumor cells. A few studies on large groups of patients disproved of this premise, demonstrating a similar healing rate between the group receiving and the one without additional treatment with dexrazoxane (6). At the same time, the hypothesis that the use of this agent increas- 
es the risk of a secondary malignancy was contradicted (8).

Several angiotensin converting enzyme inhibitors were taken into consideration, such as valsartan, captopril, and enalapril (6), which reduce the cardiotoxic effects induced by doxorubicin in adult oncological patients, without significantly influencing blood pressure. At the same time, zofenoprilat appears to be useful in this context (52). For the time being, however, there are no data on the correct dosage, nor the optimal time of administration, either concomitantly with the AC dose or a month apart (8).

Statins are used in patients with atherosclerotic disease, having a reducing effect on lipids, anti-inflammatory and antioxidant. According to studies on this subject, statins may represent agents capable of decreasing the incidence of CIC (28). Lovastatin, Fluvastatin, and atorvastatin were analyzed for this purpose (22).

Carvedilol and nebivolol are adrenergic antagonists used in the treatment of cardiovascular diseases, which demonstrated properties of antioxidant and reducing agents of CIC in preclinical studies (8).

Probucol is an antioxidant and a lipid reducer, which has been studied in mice. It appears to decrease the risk of atherosclerotic disease without influencing the cancerous effect of doxorubicin. Some studies have shown that mice which did not receive Probucol before AC administration presented a lower level of an antioxidant enzyme, GSHPx, or a superoxide-dismutase, and another analysis showed the protective activity of probucol against thiobarbituric acid, an oxidative stress marker (6).

$\mathrm{N}$-Acetylcysteine is a mucolytic agent with anti-cardiogenic and anti-genotoxic properties, which is considered a potential cardioprotective agent (6).

Sildenafil is a phosphodiesterase inhibitor used in the treatment of pulmonary hypertension in children with congenital heart disease. Its cardioprotective effect has been studied in adults treated with chemotherapy with very promising results (6).

Adiponectin is a hormone involved in various metabolic processes. Studies on mice highlighted its potential to reduce CIC (53).

Erythropoietin is produced in kidneys, but it is also found in other tissues, such as cardiac, cerebral, muscular, and spinal tissue. A decrease in CIC incidence was shown on mice receiving doxorubicin and erythropoietin concomitantly (6).

Amifostine is a cytoprotective phosphatase, which seems to have an effect against CIC, at least in preclinical studies (6).

A flavonoid, monohydroxyethylrutoside, showed promising results on mice, but human patients are still under investigation (9).

Rimonabant is a cannabinoid receptor antagonist used in obese patients setting (54) which seems to have cardioprotective effects by interfering with some mechanisms of doxorubicin (9).

In addition to these pharmacological agents, other strategies for reducing CIC incidence have also been studied. Therefore, although mild food restrictions may diminish the risk of cardiac impairment, a specific dietary regimen for these patients has not yet been established. There is currently no consensus on the benefits offered by physical exercise (20).

\section{Conclusions}

Despite the efforts to limit the incidence of treatment-related cardiotoxicity by developing protocols involving the use of few AC doses, but also by simultaneous cardioprotective agents, cardiac impairment induced by oncological treatment remains an important problem among pediatric cancer survivors. 


\begin{abstract}
Abbreviations
AC - anthracyclines

ALL - acute lymphoblastic leukemia; BFM-AL IC - Berlin-Frankfurt-Munster Intercontinental Study Group for acute lymphoblastic leukemia BNP - natriuretic peptide type B

$\mathrm{CD}$ - cumulative dose

CIC - chemotherapy-induced cardiotoxicity

DNA - deoxyribonucleic acid

FDA - Food and Drug Administration

H-FABP - heart-specific fatty acid binding protein

LVEF - left ventricle ejection fraction

HSCT - hematopoietic stem cells transplant

PCR - polymerase chain reaction

RNA - ribonucleic acid

RT-PCR - reverse transcriptase polymerase chain reaction

Top - topoisomerase
\end{abstract}

\section{Authors' contributions}

RLE (conceptualization, methodology, data curation, investigation, formal analysis, project administration, writing-original draft). RCS (writing-review and editing). ACV (conceptualization, methodology, data curation, formal analysis, writing-review and editing, supervision). CA (conceptualization, methodology, data curation, formal analysis, visualization, writing-review and editing, supervision).

\section{Conflict of interest}

None to declare.

\section{References}

1. Imbach $P$, Kuhne $T$, Arceci RJ. Introduction: Incidence and management of childhood cancer. In Imbach P, Kuhne T, Arceci RJ (editors). Pediatric Oncology, a comprehensive guide. Springer-Verlag Berlin Heidelberg. 2011; XVII-IX DOI: 10.1007/978-3-642-20359-6

2. Sági JC, Egyed B, Kelemen A, Kutszegi N, Gezsi A,
Herlitschke MA, et al. Possible roles of genetic variations in chemotherapy related cardiotoxicity in pediatric acute lymphoblastic leukemia and osteosarcoma. BMC Cancer. 2018; 18(1):704 DOI: 10.1186/s12885018-4629-6

3. Spector LG, Ross JA, Robison LL, Bhatia S. Chapter 3. Epidemiology and etiology. In Pui CH (editor). Childhood Leukemias. Cambridge University Press, New York, USA. 2006; 48-66 DOI: 10.1017/ CBO9780511471001.004

4. Silverman LB. Chapter 10: Acute lymphoblastic leukemia. In Orkin SH, Fisher DE, Look AT, Lux SE, Ginsburg D, Nathan DG (editors). Oncology of Infancy and Childhood. Elsevier, Philadelphia, USA. 2009; $297-$ 330 DOI: 10.1016/B978-1-4160-3431-5.00010-8

5. Reulen RC, Winter DL, Frobisher C, Lancashire ER, Stiller CA, Jenney ME, et al. Long-term cause-specific mortality among survivors of childhood cancer. JAMA. 2010; 304:172-9 DOI: 10.1001/jama.2010.923

6. Harake D, Franco VI, Henkel JM, Miller TL, Lipshultz SE. Cardiotoxicity in childhood cancer survivors: strategies for prevention and management. Future Cardiol. 2012; 8(4):647-70 DOI: 10.2217/fca.12.44

7. Smith OP, Hann IM. Chapter 20. Clinical features and therapy of lymphoblastic leukemia. In Arceci RJ., Hann IM, Smith OP (editors). Pediatric Hematology. Blackwell Publishing, Massachusettes, USA. 2006; 450-81 DOI: 10.1002/9780470987001.ch20

8. Christenson ES, James T, Agrawal V, Park BH. Use of biomarkers for the assessment of chemotherapy-induced cardiac toxicity. Clin Biochem. 2014; 48(0):22335 DOI: 10.1016/j.clinbiochem.2014.10.013

9. Mitry MA, Edwards JG. Doxorubicin induced heart failure: Phenotype and molecular mechanisms. Int J Cardiol Heart Vasc. 2015; 10:17-24 DOI: 10.1016/j. ijcha.2015.11.004

10. Wang GX, Wang YX, Zhou XB, Korth M. Effects of doxorubicinol on excitation-contraction coupling in guinea pig ventricular myocytes. Eur J Pharmacol. 2001; 423:99-107 DOI: 10.1016/S0014-2999(01)01096-2

11. Van der Pal HJ, Van Dalen EC. High risk of symptomatic cardiac events in childhood cancer survivors. J Clin Oncol. 2012; 30(13):1429-37 DOI: 10.1200/ JCO.2010.33.4730

12. Radu LE, Beldiman A, Ghiorghiu I, Oprescu A, Arion C, Colita A. The use of biomarkers in detecting subclinical cardiotoxicity in doxorubicin-based treatment for 
paediatric patients with acute lymphoblastic leukaemia. Rev Rom Med Lab. 2017; 25(2):157-64 DOI: 10.1515/ rrlm-2017-0014

13. Moazeni S, Cadeiras M, Yanh EH, Deng MC, Nguyen KL. Anthracycline induced cardiotoxicity: biomarkers and „Omics” technology in the era of patient specific care. Clin Transl Med. 2017; 6:17 DOI: 10.1186/ s40169-017-0148-3

14. Pizzino F, Vizzari G, Qamar R, Bomzer C, Carerj S, Zito $\mathrm{C}$, et al. Multumodality Imaging in Cardiooncology. J Oncol. 2015; 11:263950 DOI: 10.1155/2015/263950

15. Speyer SL, Kobrinsky B, Ewer MS. Chapter 63: Cardiac effects of cancer therapy. In Abeloff MD, Armitage JO, Niederhuber JE, Kastan M, Mckenna WG (editors). Abeloff's Clinical Oncology, 4th Edition. Elsevier, Philadelphia, USA. 2013; 983-96

16. Gillespie HS, McGann CJ, Wilson BD. Noninvasive diagnosis of chemotherapy related cardiotoxicity. Curr Cardio Rev. 2011; 7:234-44 DOI: 10.2174/157340311799960672

17. Lipshultz SE, Rusconi P, Scully RE. Chapter 18: assessment of cardiotoxicity during anti-cancer therapy. In Januzzi JL, Bayes-Genis A (editors). NT-proBNP as a Biomarker in Cardiovascular Diseases. Prous Science SA, Barcelona, Spain. 2007; 193-8

18. Vejpongsa P, Yeh ETH. Prevention of anthracycline-induced cardiotoxicity: challenges and opportunities. J Am Coll Cardiol. 2014; 64(9):938-45 DOI: 10.1016/j. jacc.2014.06.1167

19. Lipshultz SE, Lipsitz SR, Kutok JL, Miller TL, Colan SD, Neuberg DS, et al. Impact of hemochromatosis gene mutations on cardiac status in doxorubicin-treated survivors of childhood high-risk leukemia. Cancer. 2013; 119(19):3555-62 DOI: 10.1002/cncr.28256

20. Gianni L, Herman EH, Lipshultz SE, Minotti G, Sarvazyan N, Sawyer DB. Anthracycline cardiotoxicity: From Bench to bedside. J Clin Oncol. 2008; 26(22):3777-84 DOI: 10.1200/JCO.2007.14.9401

21. Huang C, Zhang X, Ramil JM, Rikka S, Kim L, Lee Y, et al. Juvenile exposure to anthracyclines impairs cardiac progenitor cell function and vascularization resulting in greater susceptibility to stress-induced myocardial injury in adult mice. Circulation. 2010; 121:675-83 DOI: 10.1161/CIRCULATIONAHA.109.902221

22. Hahn VS, Lenihan DJ, Ky B. Cancer therapy-induced cardiotoxicity: basic mecanisms and potential cardioprotective therapies. J Am Heart Assoc. 2014; 3:e000665 DOI: 10.1161/JAHA.113.000665

23. Blanco JG, Sun CL, Landler W, Chen L, Esparza-Duran $\mathrm{D}$, Leisenring W, et al. Anthracycline-related cardiomyopathy after childhood cancer: role of polymorphisms in carbonyl reductase genes--a report from the Children's Oncology Group. J Clin Oncol. 2012; 30(13):1415-21 DOI: $10.1200 / J C O .2011 .34 .8987$

24. Fulbright JM. Review of Cardiotoxicity in Pediatric Cancer Patients: During and after Therapy. Cardiol Res Pract. 2011; 942090 DOI: 10.4061/2011/942090

25. Galderisi M, Marra F, Esposito R, Lomoriello VS, Pardo M, de Divitiis O. Cancer therapy and cardiotoxicity: The need of serial Doppler echocardiography. Cardiovasc Ultrasound. 2007; 5:4 DOI: 10.1186/1476-71205-4

26. Martins de Souza D. Is the word „biomarker” being properly used by proteomics research in neuroscience? Eur Arch Psychiatry Clin Neurosc. 2010; 260:561-2 DOI: $10.1007 / \mathrm{s} 00406-010-0105-2$

27. Horacek JM, Vasatova M, Pudil R, Tichy M, Zak P, Jakl $\mathrm{M}$, et al. Biomarkers for the early detection of anthracycline-induced cardiotoxicity: current status. Biomed Pap Med Fac Univ Palacky Olomouc Czech Repub. 2014; 158(4):511-7 DOI: 10.5507/bp.2014.004

28. Sheppard RJ, Berger J, Sebag IA. Cardiotoxicity of cancer therapeutics: current issues in screening, prevention and therapy. Frontiers in Pharmacology. 2013; 4:19 DOI: 10.3389/fphar.2013.00019

29. De Lemos JA. Increasingly sensitive assays for cardiac troponins: a review. Jama. 2013; 309(21):2262-9 DOI: 10.1001/jama.2013.5809

30. Oztarhan K, Guler S, Aktas B, Arsian M, Salcioglu $\mathrm{Z}$, Aydogan G. The value of echocardiography versus cardiac troponin i levels in the early detection of anthracycline cardiotoxicity in childhood acute leukemia: Prospective evaluation of a 7-year-long clinical follow-up. Pediatr Hematol Oncol. 2011; 28(5):380-94 DOI: $10.3109 / 08880018.2011 .563772$

31. Cardinale D, Sandri MT, Colombo A, Colombo N, Boeri M, Lamantia G, et al. Prognostic value of troponin I in cardiac risk stratification of cancer patients undergoing high-dose chemotherapy. Circulation. 2004; 109(22):2749-54 DOI: 10.1161/01. CIR.0000130926.51766.CC

32. Lee HS, Son CB, Shin SH, Kim YS. Clinical correlation between brain natriuretic peptide and anthracycline-induced cardiac toxicity. Cancer Res Treat. 2008; 
40(3):121-6 DOI: 10.4143/crt.2008.40.3.121

33. Soker M, Kervancioglu M. Plasma concentration on NT-pro-BNP and cardiac troponin-I in relation to doxorubicin-induced cardiomyopathy and cardiac function in childhood malignancy. Saudi Med J. 2005; 26(8):1197-202

34. Specchia G, Buquicchio C, Pansini N, di Serio F, Liso V, Pastore D, et al. Monitoring of cardiac function on the basis of serum troponin I levels in patients with acute leukemia treated with anthracyclines. J Lab Clin Med. 2005; 145(4):212-20 DOI: 10.1016/j.lab.2005.02.003

35. Tian S, Hirshfield KM, Jabbour SK, Toppmeyer D, Haffty B, Gm Khan AJ, et al. Serum Biomarkers for the Detection of Cardiac Toxicity after Chemotherapy and Radiation Therapy in Breast Cancer Patients. Front Oncol. 2014; 4:277 DOI: 10.3389/fonc.2014.00277

36. Romano S, Fratini S, Ricevuto E, Procaccini V, Stifano G, Mancini M, et al. Serial measurements of NT-proBNP are predictive of not-high-dose anthracycline cardiotoxicity in breast cancer patients. Br J Cancer. 2011; 105(11):1663-8 DOI: 10.1038/bjc.2011.439

37. Cil T, Kaplan AM, Altintas A, Akin AM, Alan S, Isikdogan A. Use of $\mathrm{N}$-terminal pro-brain natriuretic peptide to assess left ventricular function after adjuvant doxorubicin therapy in early breast cancer patients: a prospective series. Clin Drug Investig. 2009; 29(2):131-7 DOI: 10.2165/0044011-200929020-00007

38. Feola M, Garrone O, Occelli M, Francini A, Biggi A, Visconti G, et al. Cardiotoxicity after anthracycline chemotherapy in breast carcinoma: effects on left ventricular ejection fraction, troponin I and brain natriuretic peptide. Int J Cardiol. 2011; 148(2):194-8 DOI: 10.1016/j.ijcard.2009.09.564

39. Garrone O, Crosetto N, lo Nigro C, Carzeddu T, Vivenza D, Monteverde M, et al. Prediction of anthracycline cardiotoxicity after chemotherapy by biomarkers kinetic analysis. Cardiovasc Toxicol. 2012; 12(2):135-42 DOI: $10.1007 /$ s12012-011-9149-4

40. El-Shitany NA, Tolba OA, El-Shanshory MR, El-Hawary EE. Protective effect of carvedilol on adriamycin-induced left ventricular dysfunction in children with acute lymphoblastic leukemia. J Card Fail. 2012; 18(8):607-13 DOI: 10.1016/j.cardfail.2012.06.416

41. Xu XY, Huang MR, Tang JY, Zhang YQ, Wu YR, Zhou M. Evaluation of early monitoring of cardiotoxicity induced by anthracyclines. Zhongguo Dand Dai Er Ke Za Zhi. 2011; 13(6):490-4
42. Sawaya H, Sebag IA, Plana JC, Januzzi JL, Ky B, Cohen $\mathrm{V}$, et al. Early detection and prediction of cardiotoxicity in chemotherapy-treated patients. Am J Cardiol. 2011; 107(9):1375-80 DOI: 10.1016/j.amjcard.2011.01.006

43. Kang Y, Xu X, Cheng L, Li L, Sun M, Chen H, et al. Two-dimensional speckle tracking echocardiography combined with high-sensitive cardiac troponin $\mathrm{T}$ in early detection and prediction of cardiotoxicity during epirubicine-based chemotherapy. Eur J Heart Fail. 2014; 16(3):300-8 DOI: 10.1002/ejhf.8

44. ElGhandour AH, Sorady ME, Azab S, ElRahman M. Human heart-type fatty acid-binding protein as an early diagnostic marker of doxorubicin cardiac toxicity. Hematol Rev. 2009; 1(1):e6 DOI: 10.4081/hr.2009.e6

45. Sayed-Ahmed MM, Al-Shabanah OA, Hafez MM, Aleisa AM, Al-Rejale SS. Inhibition of gene expression of heart fatty acid binding protein and organic cation-carnitine transporter in doxorubicin cardiomyopathic rat model. Eur J Pharmacol. 2010; 640:143-9 DOI: 10.1016/j.ejphar.2010.05.002

46. Ozturk G, Tavil B, Ozguner M, Ginis Z, Erden G, Turic B, et al. Evaluation of Cardiac Markers in Children Undergoing Hematopoietic Stem Cell Transplantation. J Clin Lab Anal. 2015; 29(4):259-62 DOI: 10.1002/ jcla.21760

47. Horacek JM, Vasatova M, Tichy M, Pudli R, Jebavy L, Maly J. The use of cardiac biomarkers in detection of cardiotoxicity associated with conventional and highdose chemotherapy for acute leukemia. Exp Oncol. 2010; 32(2):97-9

48. Lai R, Wang X, Zhang X, Lin WQ, Rong TH. Heart fatty acid-binding protein may not be an early biomarker for anthracycline-induced cardiotoxicity in rabbits. Med Oncol. 2012; 29(3):2303-8 DOI: 10.1007/s12032011-9843-x

49. Ky B, Putt M, Sawaya H, French B, Januzzi JL Jr, Sebag IA, et al. Early increases in multiple biomarkers predict subsequent cardiotoxicity in patients with breast cancer treated with doxorubicin, taxanes, and trastuzumab. J Am Coll Cardiol. 2014; 63(8):809-16 DOI: 10.1016/j.jacc.2013.10.061

50. Fichtlscherer S, Zeiher AM, Dimmeler S. Circulating microRNAs: biomarkers or mediators of cardiovascular diseases? Arterioscler Thromb Vasc Biol. 2011; 31(11):2383-90 DOI: 10.1161/ATVBAHA.111.226696

51. Lipshultz SE, Scully RE, Lipsitz SR, Sallan SE, Silverman LB, Miller TL, et al. Assessment of dexrazoxane 
as a cardioprotectant in doxorubicin-treated children with high-risk acute lymphoblastic leukaemia: longterm follow-up of a prospective, randomised, multicentre trial. Lancet Oncol. 2010; 11(10):950-61 DOI: 10.1016/S1470-2045(10)70204-7

52. Monti M, Terzuoli E, Ziche M, Morbidelli L. The sulphydryl containing ACE inhibitor Zofenoprilat protects coronary endothelium from Doxorubicin-induced apoptosis. Pharmacol Res. 2013; 76:171-81 DOI: 10.1016/j. phrs.2013.08.003
53. Konishi M, Haraguchi G, Ohigashi H, Ishihara R, Salto K, Nakano Y, et al. Adiponectin protects against doxorubicin-induced cardiomyopathy by anti-apoptotic effects through AMPK upregulation. Cardiovasc Res. 2011; 89(2):309-19 DOI: 10.1093/cvr/cvq335

54. Viner RM, Hsia Y, Tomsic T, Wong I. Efficacy and safety of anti-obesity drugs in children and adolescents: systematic review and meta-analysis. Obes Rev. 2010; 11:593-602 DOI: 10.1111/j.1467-789X.2009.00651.X 